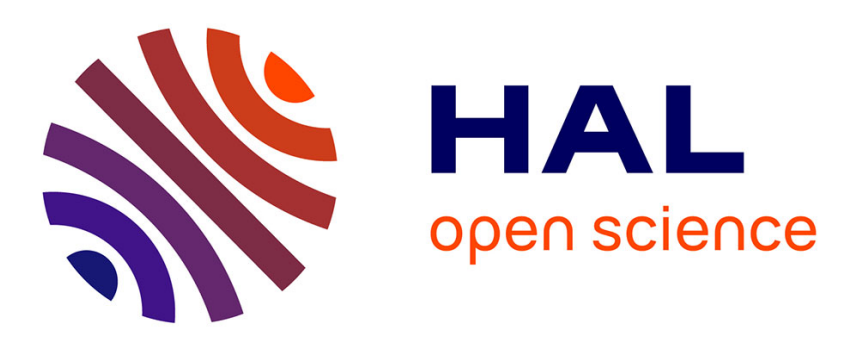

\title{
Scan-free optical correlation techniques: history and applications to optical coherence tomography
}

\author{
L. Froehly, R. Leitgeb
}

\section{To cite this version:}

L. Froehly, R. Leitgeb. Scan-free optical correlation techniques: history and applications to optical coherence tomography. Journal of Optics, 2010, 12 (8), pp.084001. 10.1088/2040-8978/12/8/084001 . hal-00517800

\section{HAL Id: hal-00517800 \\ https://hal.science/hal-00517800}

Submitted on 30 Apr 2021

HAL is a multi-disciplinary open access archive for the deposit and dissemination of scientific research documents, whether they are published or not. The documents may come from teaching and research institutions in France or abroad, or from public or private research centers.
L'archive ouverte pluridisciplinaire HAL, est destinée au dépôt et à la diffusion de documents scientifiques de niveau recherche, publiés ou non, émanant des établissements d'enseignement et de recherche français ou étrangers, des laboratoires publics ou privés. 


\title{
Scan-free optical correlation techniques: history and applications to optical coherence tomography
}

\author{
Luc Froehly $^{1}$ and Rainer Leitgeb ${ }^{2}$ \\ ${ }^{1}$ Institut FEMTO-ST, UMR CNRS 6174, Université de Franche-Comté, \\ 25030 Besançon Cedex, France \\ ${ }^{2}$ Medical University of Vienna, Centre for Biomedical Engineering and Physics, Vienna, \\ Austria \\ E-mail: luc.froehly@univ-fcomte.fr
}

\begin{abstract}
In parallel with progress in generating ultrafast pulse sources and characterization techniques, optical time correlation techniques have seen tremendous development over many years and paved the way for novel applications in non-destructive and high resolution 'optical coherence tomography' (OCT) imaging. Amongst the known correlation techniques, the scan-free approach presents the advantage of single shot detection and real-time acquisition for pulse measurements, but this is not generally considered and applied for OCT imaging. The aim of this paper is to review the scan-free correlation method, analyze its performance and extended features and discuss its application to OCT.
\end{abstract}

Keywords: optical coherence tomography, scan-free, optical correlation

\section{Introduction}

Ultrashort laser pulses are of particular interest to generate, measure and analyze dynamic processes in biological media [1]. The development of the high resolution time of flight optical correlation technique has occurred over nearly three decades, advancing in parallel with the shortening of laser generated light pulses. Indeed, with sub-picosecond light pulses the bandwidth of electronic detection techniques is not sufficient to fully and accurately characterize the phase and amplitude of ultrashort pulses [2] and other optical approaches need to be considered. Time of flight measurement setups are generally based on optical correlation together with a nonlinear interaction of the light into a specific medium. This leads to an intensity correlation of the fields instead of an amplitude correlation [3].

Optical correlators involve splitting of the incoming beam and a delay line to obtain different delays between the two arms in order to measure a correlation signal. Much work has been done in this field and an extensive review of the techniques developed has been given in [3] by Trebino, the co-inventor of one of the most celebrated systems of pulse measurement-FROG (frequency resolved optical gating) [4]. Since there is an obvious relationship between pulse time of flight and pulse path length, it is not surprising that optical correlation techniques for pulse characterization have led to new approaches to imaging through biological media. Indeed, a few authors already known in the field of laser developments and pulse shape measurements have contributed to the application of time correlation to the field of biological imaging through the technique now known as optical coherence tomography (OCT) $[5,6]$. The basic elements of OCT are very similar to echographic imaging: a short light pulse (as viewed by the detection system, i.e. an interferometer which is only sensitive to the power spectral density of the incoming light) is incident on the medium to image, and then 'light echoes' that are backscattered by the medium are detected by an optical time of flight measurement system. Sample impulse response can not be directly registered by electronic detection for the same reason as for pulse measurements: the electronic bandwidth is too low. 
The first and most well established method is time domain OCT (TDOCT) [5, 7-9]. This method is simply based on a linear optical correlator with mechanical scanning (e.g. a Michelson interferometer). However, in the context of using optical correlators for pulse characterization, there is a case where mechanical scanning is unsuitable. Specifically, when one wants to measure single shot events or non timestable pulses, a 'scan-free' system should be used to realize autocorrelation pulse per pulse. This particular case has led the scientific community to develop 'scan-free' linear correlators [3, 11, 12]. The OCT community has also benefited from these 'scan-free' systems, allowing access to real-time information. This is the case of the so called 'Fourier domain OCT (FDOCT)', where there is no need for mechanical scanning to measure correlation as the signal is registered in the spectral domain. Apart from those two main categories of OCT systems, there is also the lesser known 'time domain scan-free optical correlator' (numerous other names can be found in the literature), which basically is very similar to single shot FROG systems but in a linear regime. Whereas TDOCT and FDOCT are widely described in the literature through numerous books, reviews and publications, the scan-free time domain approach has not to date been the subject of extensive review. Our aim here is thus to give a comprehensive overview of history, performance, and possibilities of this kind of system by summarizing the existing literature. The paper is organized as follows.

(i) In section 2 we will give a short overview of OCT technology through its two main methods, TDOCT and FDOCT.

(ii) Section 3 will be devoted to various reports of the 'time domain scan-free optical correlator' appearing in the literature. The history of the system through different fields in optics, its main principles and its performance and characteristics compared to other systems will be given particular emphasis.

(iii) Finally we conclude in section 4 about possibilities of such scan-free time domain devices to be used in the context of OCT.

\section{OCT in general}

\subsection{OCT performance-definitions}

Before giving a short overview of OCT principles, we first introduce the main parameters generally used to describe the performance of OCT systems.

- Spatial resolution: this means, (as with every imaging system) the minimum size pattern we can distinguish laterally. The lateral resolution in OCT is exactly, as in microscopy, set by the optical properties of the microscope objective which illuminates the sample. In the paraxial approximation the resolution $\Delta x$ is given by

$$
\Delta x=\frac{\alpha \lambda f}{d}
$$

where $\alpha$ is a constant depending on the shape of the focused beam: Gaussian, Lorentzian, Bessel beam or anything else. $\lambda$ is the beam central wavelength, $f$ the objective lens focal length and $d$ the beam diameter on the objective lens.

- Axial resolution: the axial, or depth, resolution in OCT is determined by the light beam coherence length. For a Gaussian spectrum the axial resolution $\delta z$ is written

$$
\delta z=\frac{2 \ln 2 \cdot \lambda^{2}}{\pi \cdot \Delta \lambda}
$$

where $\Delta \lambda$ is the optical bandwidth of the light beam.

- Acquisition speed: this is basically the speed at which images are acquired. As in OCT there can be different kinds of images, depending essentially on the application, it has to be defined precisely.

* A-scan: this means that the image is a line in the sample. For a fixed $x, y$ position $z$ is made variable.

* B-scan: a B-scan is a set of A-scans, i.e. a plane in the sample. For a given $x$ (or $y$ ) all $y$ (respectively $x$ ) and $z$ are acquired.

* Volume: this is a set of B-scans which finally gives a $3 \mathrm{D}$ volume image.

- Sensitivity: the sensitivity sees different definitions in the literature. Receiver sensitivity is defined, for example, as the signal which corresponds to a given signal to noise ratio (SNR) [13]. But in the case of an optical reflectometer it does not mean the same thing. In this case, the sensitivity is defined as the maximum value of the SNR, that is when the reflectivity of the sample is equal to 1 [14]. In OCT different definitions have been given but these definitions begin to converge to one which can be expressed as 'the lowest reflectivity that can be detected by the system' [15]. In the case of a shot-noise limited detection system the sensitivity $S$ can then read

$$
S=10 \log \left(\frac{\eta P_{\mathrm{s}}}{2 h v_{0} B}\right)
$$

where $\eta$ is the quantum efficiency of the detector, $P_{\mathrm{s}}$ the incoming power onto the sample, $h$ the Planck constant, $v_{0}$ the light average frequency and $B$ the detection bandwidth. This equation simply shows that when an OCT system is shot-noise limited the system is almost able to detect a single backscattered photon per detection cell. This explains the tremendous sensitivity of OCT systems.

\subsection{TDOCT}

The history of TDOCT probably began in 1972 with Flournoy and his low coherence interferometry system for thickness gauge measurement [16]. This kind of system was later called optical (low-) coherence reflectometry (OLCR) and used for determining positions and magnitudes of reflection sites within miniature optical assemblies for example [17]. First approaches based on OLCR by Fercher and Hitzenberger [7, 8] imaged topographic profiles of the human ocular fundus. Later on Huang [5] presented in vitro tomograms of human retina using a fiber-based system introducing the notion of OCT. In 1993 Fercher [9] and Swanson [10] published in vivo images of 


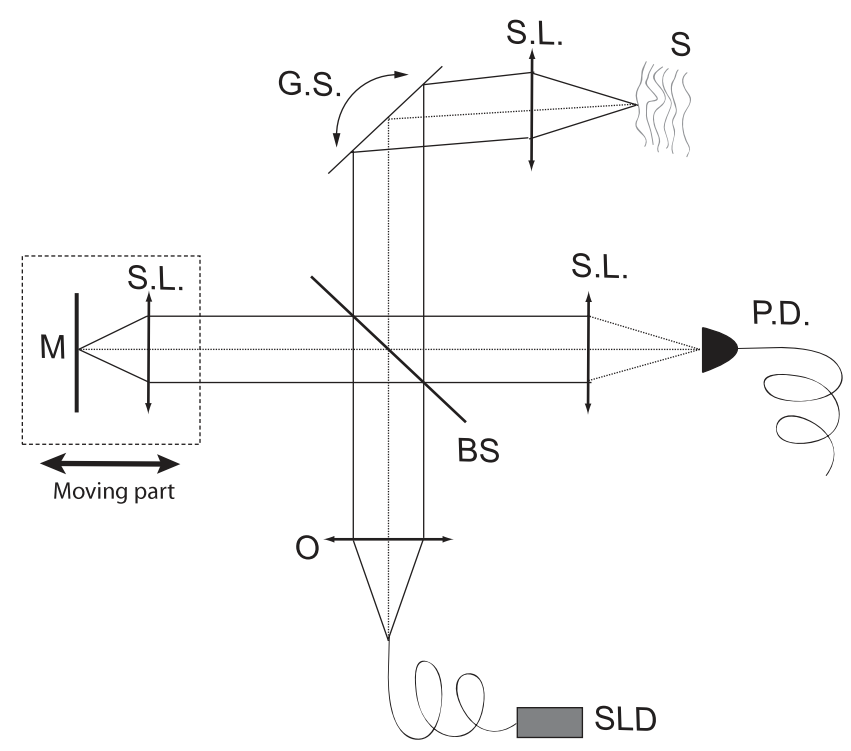

Figure 1. Classical setup for TDOCT: SL, spherical lens; O, microscope objective; SLD, superluminescent photodiode; BS, beam splitter; GS, galvano scanning mirror; PD, photodiode; $\mathrm{M}$, mirror; S, sample.

human retina. The application to a diffusive biological tissue with the claim of the real potential of OLCR to discriminate light reflected from a small volume in a sample was also revealed by Clivaz [18] almost simultaneously. All these systems are based on linear time domain correlators with a mechanical scanning unit to record the time correlation (see figure 1). A basic equation for TDOCT gives the registered intensity as a function of scanning time $t$ as

$$
I(t)=\int|e(t)|^{2} \mathrm{~d} t+\int|s(t)|^{2} \mathrm{~d} t+2 \mathcal{R} e\{e(t) \otimes s(t)\}
$$

where $|e(t)|$ is the modulus (normally $e(t)$ is chosen to be real so that $|e(t)|=e(t))$ of the reference arm signal, $|s(t)|$ is the modulus of the signal coming back from all scattering layers of the sample, $\mathcal{R} e$ is the real part and $\otimes$ denotes the correlation operation. A few papers have also used nonlinear correlators, directly based on pulse measurement techniques. The setup of this nonlinear OTDR (optical time domain reflectometry) system was described first by Fontaine in 1981 [19] using subpicosecond light pulses. Fujimoto demonstrated, a few years later, the application to biological imaging using femtosecond pulses [20]. However, there is currently no commercial nonlinear correlator for biomedical imaging; the reason is probably the energy needed to produce such nonlinear effects in materials and also the cost of nonlinear crystals together with the difficulty of alignment for such a system (phase matching conditions).

We now summarize the best performance of TDOCT systems until now.

- A $1 \mu \mathrm{m}$ depth resolution in vivo has been reported since 1999 [21]. This needs ultra-broadband and bright light sources like femtosecond laser sources or supercontinuum laser sources [22].
- The acquisition speed has reached its current limit with the use of the grating based rapid scanning delay line introduced first in 93 in an autocorrelator for pulse shape measurements [23]. Such delay lines allow an A-scan acquisition rate as high as $2 \mathrm{kHz}$ with a depth scanning range of $3 \mathrm{~mm}$ [24].

- In principle, the sensitivity of TDOCT systems can reach more than $100 \mathrm{~dB}$ as it only depends on the detection bandwidth $B$ and the power incident onto the sample $P_{\mathrm{s}}$ (see section 2.1). With a rapid scanning delay line a $106 \mathrm{~dB}$ sensitivity has been reached in vivo with an A-scan rate of $2 \mathrm{kHz}$ and a power onto the sample of $10 \mathrm{~mW}$ [25]. According to laser safety standards a power of $800 \mu \mathrm{W}$ at a center wavelength of $850 \mathrm{~nm}$ incident on the eye can be considered as safe. This clearly limits the possible imaging speeds for the eye for keeping high sensitivity.

We will not go further into details of TDOCT technology as our aim is essentially to give some main ideas about this field to be able to compare later with time domain scan-free optical correlation techniques. For the reader wishing to go further into this technique without reading the whole and extensive bibliography in the subject we recommend some references: of course the original papers introducing OCT [7, 8, 5], but also the review paper on OCT techniques and applications by Fercher [26] and finally various books [27, 28].

\subsection{FDOCT}

As seen in the previous paragraph, to get an image of an in vivo retina in a human eye with a limited power incident onto it, with a $95 \mathrm{~dB}$ sensitivity the A-scan rate can reach almost $2 \mathrm{kHz}$ in TDOCT. This means that a B-scan composed of 1000 A-scans will capture movements inside the sample at the tissue scale level. It appears then that state of the art TDOCT systems are limited when dealing with accurate biological imaging. In particular, if the aim is to make 'real-time' $(8 \mathrm{~Hz})$ volume imaging then TDOCT is not suitable. On the other hand, in 2003 Leitgeb demonstrated that FDOCT presents a $20 \mathrm{~dB}$ sensitivity gain with an increased acquisition speed with respect to TDOCT [15]. The system is apparently very similar to TDOCT but involves a spectrometer at the interferometer output so that the signal is directly measured in the spectral plane (see figure 2). A basic equation of the FDOCT signal registered at the spectrometer output is given in terms of the light frequency $v$ below:

$$
I(v)=|E(v)|^{2}+|S(v)|^{2}+2 \mathcal{R} e\left\{E(\nu) S(v) \mathrm{e}^{\mathrm{j} 2 \pi \nu \tau}\right\}
$$

where $E(v), S(v)$ are respectively the Fourier transform of $e(t), s(t)$ and $\tau$ is the time mismatch between the two unbalanced arm of the interferometer ( $\tau=\frac{d}{c}$ where $d$ is the difference in optical path between the two arms and $c$ the speed of light in vacuum). In FDOCT an inverse Fourier transform is numerically processed to retrieve the time domain signal. This leads to the following expression:

$$
\begin{aligned}
& I(t)=e(t) \otimes e(t)+s(t) \otimes s(t)+(e(t) \otimes s(t)) \\
& \star(\delta(t-\tau)+\delta(t+\tau))
\end{aligned}
$$




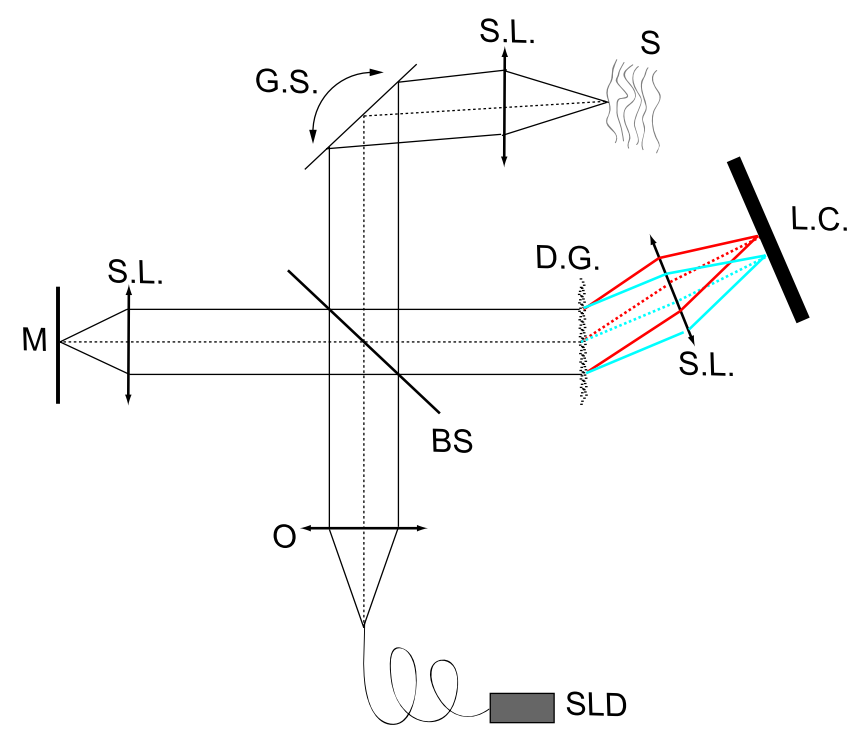

Figure 2. Classical setup for FDOCT: SL, spherical lens; O, microscope objective; SLD, superluminescent photodiode; BS, beam splitter; GS, galvano scanning mirror; LC, line detector; M, mirror; $\mathrm{S}$, sample; DG, diffraction grating.

(This figure is in colour only in the electronic version)

with $\star$ being the convolution operator and $\delta()$ being the usual Dirac distribution. The main advantage of FDOCT with respect to TDOCT is a higher sensitivity with an increased acquisition speed. The main reason has been accurately described by Leitgeb [15] and can be intuitively understood by the fact that an A-scan is obtained instantaneously on the linear detector and each pixel of the detector contains then information about all depths simultaneously. The sensitivity gain for an equivalent integration time is then about $\log (N / 4)$, where $N$ is the number of pixels of the linear detector (see for example [26] pp 69-71). As in TDOCT the detection bandwidth $B$ is given essentially by the time needed to acquire one point of the A-scan, we clearly see that we can also gain in acquisition speed in FDOCT as now the detection bandwidth is directly the A-scan rate and hence the detector frame rate. With a CMOS linear detector as fast as $100 \mathrm{kHz}$ we clearly see the tremendous gain both in sensitivity and speed of FDOCT with respect to TDOCT.

The uptake of this approach as a powerful imaging technique for biological imaging is due to Leitgeb, even though the principles of FDOCT are of course older than this. Indeed, the first attempt to image biological samples with this technique is due to Fercher [29]. The underlying white light interferometric technique has in fact been well known since 1973 [30, 31]. Spectral interferometry is actually also used in different fields of optical characterization and especially in pulse characterization $[3,32,33]$.

The main limitation of the FDOCT setup, as first developed, was essentially due to the spectrometer resolution together with the number of pixels available on the detector. Indeed, as shown in equation (6) after inverse Fourier transforming, there are three terms in the A-scan: an autocorrelation term, centered onto the zero delay, the OCT Ascan itself (positioned on $\tau$ ) and its conjugated image in $-\tau$.
Then half the number of acquired pixels is used effectively to image the sample. The limitation of the pixel size on the detector also implies a limit in the spectral resolution. This limit in spectral resolution, after Fourier transform operation, directly leads to a sensitivity decay of the system with the measurement depth [15]. Recent developments in wavelength tuning light sources showed a better performance concerning sensitivity decay, since a point detector could be used to record the instantaneous spectrum. In particular, FDML (Fourier domain mode locked) source technology offers fast sweep times of several hundred $\mathrm{kHz}$ without sacrificing output power. Still the highest sensitivity is found close to the zero delay. Then the OCT image term centered on $\tau$ has to be as close as possible to the zero delay without overlapping with the autocorrelation term. To give an example, the acquisition of an A-scan with a resolution of $1 \mu \mathrm{m}$ and a depth of field of $1 \mathrm{~mm}$ will imply almost 3000 acquisition points to fulfill Shannon criteria and no less than 6000 pixels to take into account the fact that half the number of pixels is lost. Several works have been done to remove the 'depth ambiguity' in order to benefit from the full depth range and also from the maximum sensitivity range. All methods are based on the retrieval of the complex signal spectrum which has been lost through quadratic detection. To achieve such a phase retrieval an additional reference phase shift is produced and registered using different kinds of optical setups to modulate the optical path in the reference arm of the interferometer [34, 35, 37] as usually realized in phase shifting interferometry. The extraction of phase information and the ambiguity removal can be then achieved through different algorithms: phase shifting algorithms (involving two or up to five phase steps) [34, 39] or Hilbert transform methods and similar signal processing [35, 36, 38, 40-42]. Generally speaking, all these methods give rise to interesting performance in terms of artifact removal but they also all encounter some limitations that we will try to briefly summarize.

- Techniques involving piezoelectric actuators or electrooptic or acousto-optic modulators are quite complex and/or expensive systems, with difficult synchronization issues which lead to further difficulties in integrating the systems.

- Phase shifting algorithms are highly sensitive to any phase mismatch (sample movements, scanning nonlinearity), producing residual artifacts. There is also a difficulty in producing an achromatic phase shift (using inexpensive techniques) when a broadband spectrum is used.

- The quickest, cheapest, and most efficient systems are probably those based on Hilbert-like processing with transverse scanning or reference wavefront tilting [35, 40, 42]. Such systems should lead indeed to real-time acquisition of 'artifact-free' A-scans or even B-scans (Bu paper; [42]). In fact, these systems also encounter limitations due to phase fluctuations and/or the spatial fluctuations of the sample in the lateral dimension. These parameters influence directly the spatial frequency spectrum width and hence the artifact removal efficiency [40]. 
Although it is clear that acquisition time itself has increased a lot with FDOCT, the image display time is not necessarily as fast. Indeed, FDOCT requires increased computation time to do first a rescaling of acquired data from wavelength to frequency and then to do Fourier transform operations. Recent work shows tremendous improvements regarding the real-time display capability [43, 44]. They use optical components (prism) to optically process the rescaling and couple the signal processing with a dedicated GPU (graphics process unit) [44]. An alternative approach is the use of field programmable gate array programming [43]. Although these techniques yield a real-time display of images, the optical rescaling is highly dependent on the wavelength range and the central wavelength used for OCT imaging, and also FGPA programming remains an expensive technique. For further understanding of FDOCT techniques the reader is referred to $[15,26,28]$.

\section{Time domain scan-free optical correlation techniques}

In section 2 we have presented the two main categories of OCT system with their inherent properties. It appears on one hand that TDOCT is limited, in terms of speed, by the axial scanning needed to get access to the correlation, but then the sensitivity is independent of the imaging depth. On the other hand FDOCT has proven to be a quicker (acquisition time) and more sensitive method but at the cost of higher numerical processing effort and a depth dependent sensitivity (which can be also reduced through further numerical processing). One natural question can be then: is there any solution to have direct registration of an A-scan in the time domain without any need of scanning to get the time correlation? Another subsequent question will also be: if such a method exists what will be the counterpart of such a system in terms of performance? This will be the subject of the following section.

\subsection{History}

Actually, the first mention in the literature of the setup used today for the time domain scan-free optical correlation technique is due to Connes [45]. This system was developed to address the problem of the computation time needed to recover the spectral data of Fourier transform spectrometry without any loss of spectral bandwidth. The system, called SISAM (spectromètre interférentiel par sélection de l'amplitude de la modulation), was composed of a Michelson interferometer with two mirrors replaced by two reflective diffraction gratings in Littrow incidence (see figure 3). The rotation of the two gratings (in the same direction and with the same angle) allows the scanning of the spectrum with a resolution close to the grating resolution, while its translation allows the optical path change, which subsequently leads to a frequency modulation of the wavelength under inspection with a fringe contrast directly dependent on the wavelength weight in the spectrum. A Mach-Zehnder implementation of this system was first used for time of flight imaging nearly 40 years later by Brun et al to measure the difference of time propagation between two

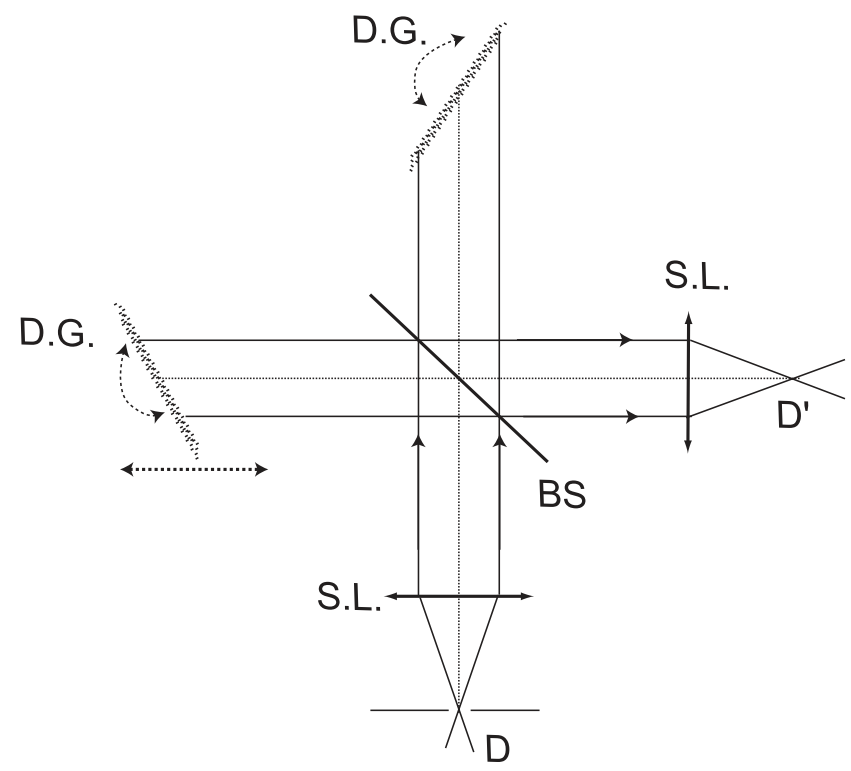

Figure 3. SISAM setup: DG, reflective diffraction gratings in Littrow incidence; BS, beam splitter; SL, spherical lens; $\mathrm{D}^{\prime}$, image of the spectrometer entrance slit D through the imaging lenses. Inspired by and redrawn from [45].

modes of a nearly monomode optical fiber [46]. Almost simultaneously, in the field of short pulse characterization, Purchase in 1993 proposed a very similar direct correlation technique for pulse imaging [11]. Similar to the idea of Purchase, in 1996 Zeylikovich proposed a modified version of the system described by Brun in 1992 to show single shot cross-correlation. A few years later Verrier and also Zeylikovich demonstrated phase object imaging through this direct correlation technique. Zeylikovich first introduced scattering in the experiment and used a single grating configuration instead of the two grating classical SISAM configuration [6, 47, 48]. In 1998 Zeylikovich proposed what can be called the first real-time B-scan image on an home-made phase object. This B-scan was obtained without any scanning part [49] thanks to a cylindrical lens introduced in the sample arm and a two-dimensional CCD detector. Watanabe in 2006 showed the first in vivo B-scan without any moving element on a biological sample. The sensitivity was $90 \mathrm{~dB}$ and the B-scan rate $10 \mathrm{~Hz}$. In 2003, Hauger demonstrated a gratingfree direct correlation OCT system fiber-based system with Bscan images onto both in vitro and in vivo samples [50]. A combination of [49] and [50] has been proposed by Koch et al, which leads to a fibered version system with down-converted fringe pattern frequency [51]. A $97 \mathrm{kHz}$ A-scan rate, $93 \mathrm{~dB}$ sensitivity, $4 \mathrm{~mm} \times 2.6 \mathrm{~mm}$ (lateral range versus axial range in the image) and $14 \mu \mathrm{m}$ axial resolution has been reached recently by Watanabe [52], which is today the state of the art with this technique. Watanabe used an InGaAs line scan camera with 512 pixels and a superluminescent diode with center wavelength $1.33 \mu \mathrm{m}$ and a full width at half-maximum spectral width of $57.6 \mathrm{~nm}$. The use of a diffraction grating together with the scan-free time domain correlation also gives rise to interesting properties that have been recently outlined by Froehly [53-55]. 
- This system is a candidate for real-time, optically processed, spectroscopic OCT [53, 54] using a dispersive element to get the spectroscopic information simultaneously with the depth information. This depth-frequency information is in fact very similar to that obtained by the Wigner transform but optically processed and registered at frame rate onto a two-dimensional CCD detector.

- High order dispersion properties of optical gratings can be used to get a direct and tunable correction of residual dispersion terms of an OCT system [55].

\subsection{Physical working principle}

3.2.1. Main principle of correlation (strongly inspired from [54] by Froehly et al). Different configurations can be found in the literature, but the general principle of time domain scan-free optical correlation techniques is similar to the one depicted in figure 4. For the sake of clarity we focus here on the recombination of reference and sample arms where the temporal correlation takes place. The lens L1 images the grating plane on the CCD detector plane D. Then we have $\overline{\overline{O A}}=\gamma \approx \frac{\theta_{\mathrm{d}}}{\theta_{\mathrm{d}}^{\prime}}$ in the case of the small angle approximation where $\gamma$ is the magnification factor. This assumption is valid while the size of the detector is much smaller than the distances of the magnification system.

Two temporal signals $r(t)$ and $s(t)$ with the same polarization state are incident onto the grating $(\mathrm{R})$ with opposite angles $\theta_{i}$ and $-\theta_{i} . r(t)$ and $s(t)$ have complex spectra given by $\hat{R}(v)$ and $\hat{S}(v)$ respectively, where $\hat{R}(v)$ and $\hat{S}(v)$ are Fourier transforms of $r(t)$ and $s(t) . \hat{R}(\nu)$ and $\hat{S}(\nu)$ can be expressed with their complex form $\hat{R}(v)=R(v) \mathrm{e}^{\mathrm{j} \cdot \varphi_{R}(v)}$ and $\hat{S}(v)=S(v) \mathrm{e}^{\mathrm{j} \cdot \varphi_{S}(v)}$. The moduli of these spectra are directly accessible in the spectral plane of the spectrometer which is physically in the back focal plane of the lens L1.

Light at wavelength $\lambda_{i}$ coming from $r(t)$ and $s(t)$ respectively focuses in the spectral plane at two points that are symmetrical with respect to the optical axis. Then it produces in the detector plane $\mathrm{D}$ a classical two wave interference pattern of period $\frac{\lambda_{i}}{2 \cdot \sin \theta_{\mathrm{d}}^{\prime}}=T_{f}$, where $\theta_{\mathrm{d}}^{\prime}$ is the diffraction angle at wavelength $\lambda_{i}$. This configuration is similar to Young's experiment with the important difference that the position of the secondary sources is dependent on the wavelength because of the diffraction law. Each wavelength $\lambda_{i}$ leads to its own fringe pattern and all these patterns are summed up in intensity because of the non-coherence of fringe patterns obtained with different wavelengths. The resulting pattern is the sum over the effective spectral bandwidth of these individual 'Younglike' fringe patterns. This effective bandwidth depends on the detector spectral bandwidth, on the size of the lens L1 and on the grating dispersion power.

In the following analysis we assume that the frequency of a specific fringe pattern does not exhibit any nonlinearity (plane wave approximation). Hence the signal $C(z)$ in plane $D$ is

$$
\begin{aligned}
C(z) & =I_{0}+\int_{\nu} \hat{S}(v) \overline{\hat{R}(\nu)} \mathrm{e}^{\left(-\mathrm{j}\left(\frac{4 \pi v}{c} \sin \theta_{\mathrm{d}}^{\prime}\right) z\right)} \mathrm{d} v \\
+ & \int_{\nu} \hat{R}(v) \overline{\hat{S}(v)} \mathrm{e}^{\left(+\mathrm{j}\left(\frac{4 \pi v}{c} \sin \theta_{\mathrm{d}}^{\prime}\right) z\right)} \mathrm{d} v
\end{aligned}
$$

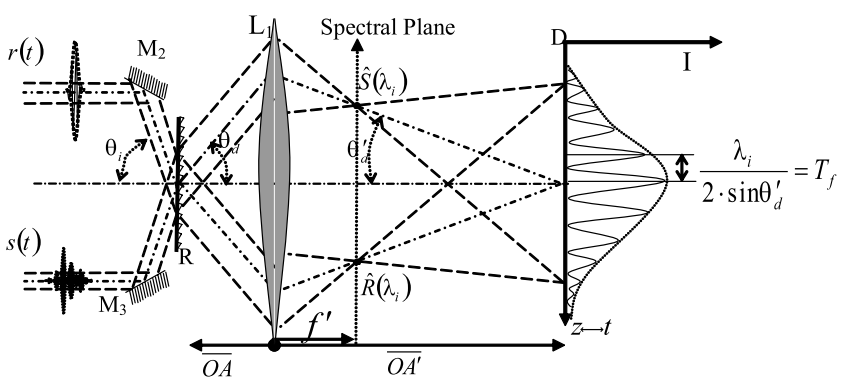

Figure 4. Correlator working principle. Extracted from [54] by Froehly et al.

where $\overline{()}$ denotes here the conjugate of the complex spectrum of the fields whereas in the previous section this notation denoted the algebraic measure. The grating relation is

$$
\sin \theta_{\mathrm{d}}-\sin \theta_{i}=-\frac{\lambda}{\Lambda}
$$

for the minus one diffraction order where $\Lambda$ is the grating periodicity. Relations (7), (8) together with the magnification relation lead to the following formulation of $C(z)$ :

$$
C(z)=I_{0}+2 \mathcal{R} e\left[\int_{\nu} \hat{R}(v) \hat{S}(v) \mathrm{e}^{-\mathrm{j} 2 \pi\left(\frac{2 z}{\gamma c} \sin \theta_{i}\right) v} \mathrm{e}^{\mathrm{j} \frac{4 \pi z}{\gamma \Lambda}} \mathrm{d} v\right]
$$

where $z$ is the horizontal coordinate on the CCD camera lines, $I_{0}$ the background intensity, $\mathcal{R} e$ designates the real part and $c$ the speed of light in vacuum. Equation (9) shows clearly the correlation operation between the temporal fields realized by the system. The temporal variable is spatially displayed through the variable change $t=\frac{z}{c}$.

\subsubsection{Grating effect. Equation (9) can be also rewritten as}

$$
C(z)=I_{0}+2 \mathcal{R} e\left[\int_{\nu} \hat{R}(v) \hat{S}(v) \mathrm{e}^{-\mathrm{j} 4 \pi\left(\frac{\sin \theta_{i} v}{\gamma}-\frac{1}{\gamma \Lambda}\right) \frac{z}{c}} \mathrm{~d} v\right]
$$

in which the term $\left(\frac{\sin \theta_{i} \nu}{\gamma}-\frac{1}{\gamma \Lambda}\right)$ is the interference pattern fringe frequency for the light frequency $v$ then $-\frac{1}{\gamma \Lambda}$ is a frequency shift of the fringe pattern, so that finally $\mathrm{e}^{\mathrm{j} \frac{4 \pi z}{\gamma \Lambda}}$ is a global fringe frequency shift which depends only on the grating parameter and on the magnification of the imaging system. Without this term we would end up with a classical correlation pattern obtained in a Young's slit experiment illuminated with a polychromatic light source. Here the spatial frequency of correlation fringes is drastically reduced with respect to Young's experiment (see for example the paper [50] which uses a Young's experiment-like setup to realize 'OCT imaging') to become compatible with the modulation transfer function of a CCD sensor. This 'Moiré-like' effect is of particular interest in terms of detection because the carrier frequency can be tuned independently of the envelope (by changing the beam incidence angle onto the grating): we then have a two scale system where the carrier has a usual phase change sensitivity of $2 \pi$ per wavelength of optical path change whereas the envelope remains approximately unchanged with a path length change sensitivity of the order of the source coherence length. 


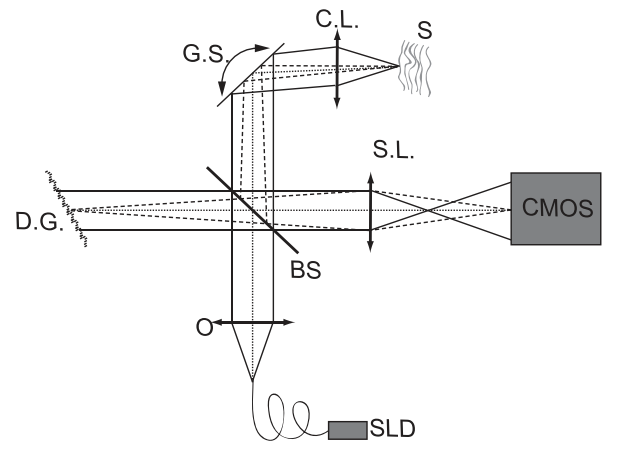

(a)

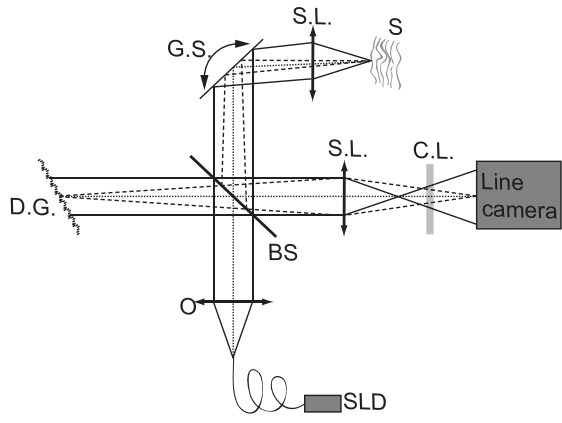

(b)

Figure 5. Setups for scan-free correlation: DG, diffraction grating; GS, scanning galvanometer; CL, cylindrical lens; S, sample; BS, beam splitter; SL, spherical lens; O, microscope objective; SLD, superluminescent diode, CMOS, CMOS 2D detector. (a) Setup for parallel imaging (frame rate B-scan); (b) setup for parallel A-scan grabbing. (Inspired by and redrawn from [56] by Watanabe $e t a l$ ).

\subsection{Performance}

Basically, time domain scan-free correlation systems based on grating correlation are inherently less sensitive, for the same incident power onto the sample and the same detector, than FDOCT systems. In fact, in both FDOCT and scanfree correlation systems the light power is divided onto the $N$ pixels of the linear detector so that the power per pixel is also divided by the same amount. We may then think that both systems would have the same sensitivity: this is nevertheless not the case, as in the FDOCT case there is a further fast Fourier transform operation which dramatically changes the distribution of the signal with respect to the noise. This results in an SNR gain in FDOCT with respect to scan-free correlation which is almost proportional to the number of pixels of the detector (i.e. $N$ ). By extension, the sensitivity of a scan-free system seems to be very similar to that of TDOCT $[28,50]$. In fact, the grating based scan-free system realizes a downconversion of the fringe pattern frequency (as seen in 3.2.2), which results in a smaller number of sampled points than in TDOCT (between one and ten times less, depending on the axial resolution of the system) and hence a corresponding sensitivity gain with respect to TDOCT for an equivalent integration time.

Despite the rather limited sensitivity of scan-free systems compared to that of FDOCT and the close sensitivity to that of TDOCT, scan-free systems present some interesting features we want to outline now.

(1) The temporal correlation is scan free, so there is no scan induced nonlinearity as can be the case in TDOCT.

(2) There is also no mirror image other than cross-correlation terms as is the case in FDOCT because the signal is directly registered in the time domain.

(3) Spectroscopic tomographic imaging can be obtained optically rather than numerically, which may enable access to spectroscopic imaging of dynamic processes [54].

(4) The use of a diffraction grating coupled to a direct time variable spatial display allows the use of high order dispersion properties of the diffraction grating for versatile and tunable dispersion compensation purposes [55].
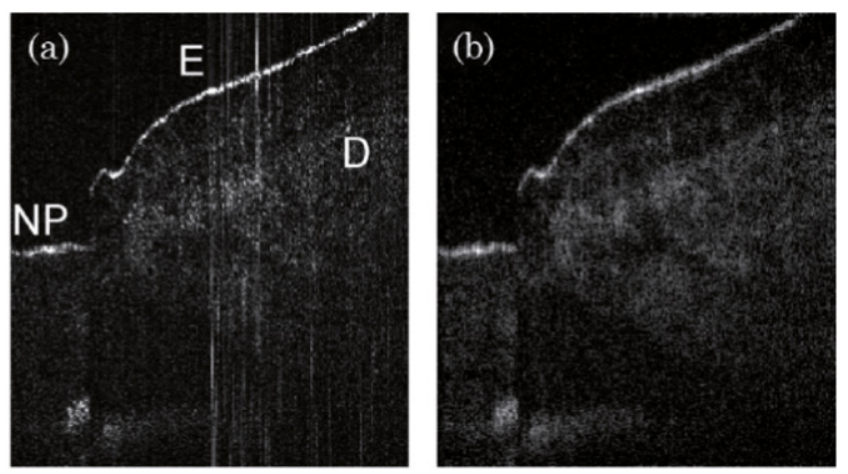

Figure 6. In vivo OCT images of a human nail-fold region by (a) the absolute value in the difference between data for two sequential lines, (b) demodulation with the Hilbert transform; NP, nail plate; E, epidermis; D, dermis; scale bar, $1 \mathrm{~mm}$. (Reprinted from Watanabe et al [52] copyright 2009, by permission of the author and the Optical Society of America.)

(5) There is no fundamental sensitivity decay with depth (as occurs in FDOCT) if the intensity of the beam on the detector remains almost constant.

Despite an admitted 15-20 dB lower sensitivity than FDOCT, scan-free correlation OCT systems have demonstrated interesting performance for fairly simple processing. Watanabe demonstrated a $76 \mathrm{~dB}$ sensitivity with a full 3D imaging of a fingerprint part in vivo at a rate of $6 \mathrm{~Hz}(5.8 \times$ $2.8 \times 2.0 \mathrm{~mm}^{3}$ [512 pixels $\times 250$ pixels $\times 512$ pixels] $)$ using a cylindrical lens to illuminate the sample so that frame rate B-scans can be acquired. The detector was a CMOS camera with $6000 \mathrm{fps}$ [56]. The setup principle is shown in figure 5(a). This kind of setup combined with an InGaAs line scan camera operating at $47 \mathrm{kHz}$ and with a spherical lens within the sample arm (see figure 5(b)) shows the possibility to acquire B-scans of in vivo fingerprint part at a rate of $94 \mathrm{fps}$ with a sensitivity of $93 \mathrm{~dB}$ [52] (see figure 6). Such performance is already sufficient to image biological samples, as demonstrated in those papers. 


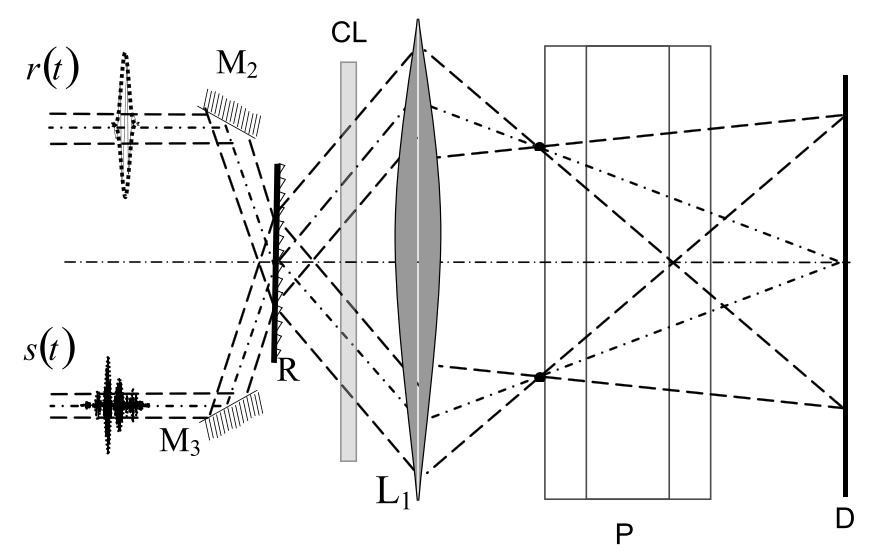

Figure 7. Configuration for optical spectro-tomography: $\mathrm{M}$, mirrors; L, spherical lens; CL, cylindrical lens; D, CCD detector; R, transmission diffraction grating, $528 \mathrm{~g} \mathrm{~mm}^{-1}$; $\mathrm{P}$, removable direct vision prism. (Reprinted from [54] by Froehly et al.)

\subsubsection{Other functionalities of scan-free correlation systems.} Spectroscopic OCT (figures and text are extracted from or inspired by [54] by Froehly et al). Spectroscopic OCT is a method which allows access to depth resolved spectral absorption. The basis of spectroscopic OCT is due to Izatt in 1997 introducing the short time Fourier transform for extracting Doppler shifts for flow quantification [57]. Several other papers presented different configurations allowing spectroscopic measurements with OCT technology. Spectroscopic OCT systems are mainly based on numerical post-processing using short time Fourier transform analysis [57, 59]. Optical post-processing of Ascans has also been studied by either differential wavelength analysis based on the use of different light sources with different central wavelength at the input [58] or using a dispersive element at the output of a TDOCT system to demultiplex continuously the A-scans obtained for each separate spectral channel and register them in parallel [60, 61]. In 2008 Froehly showed that a scan-free correlation system was also able to give at video rate a spectroscopic A-scan by simple real-time optical processing [54]. The principle is depicted in figure 7. Basically, we recognize elements of figure 4 (diffraction grating, imaging elements and detector) but with an added cylindrical lens CL and a dispersive prism P. These two elements change the detection in the plane of the 2D detector: because of this spectroscopic device, each line of the detector receives a limited bandwidth of light that can be expressed through the spectral frequency response $\hat{F}(x, v)$. Then, the correlation signal depends on the vertical coordinate on the camera and equation (9) becomes

$$
\begin{aligned}
& C(z, x)=I_{0}+2 \mathcal{R} e\left[\int_{v} \hat{F}(x, v) \hat{R}(v) \hat{S}(v) \mathrm{e}^{-\mathrm{j} 2 \pi\left(\frac{2 z}{\gamma c} \sin \theta_{i}\right) v}\right. \\
& \left.\times \mathrm{e}^{\mathrm{j} \frac{4 \pi z}{\gamma \Lambda}} \mathrm{d} v\right]
\end{aligned}
$$

where we have now the correlation in the $x$ direction and the wavelength in the $z$ direction as can be seen in figure 8 .

Numerically processed spectroscopic OCT has already proven to be quite efficient for accessing depth resolved information [62] in 'real time', so we could wonder whether there is any interest to develop such optically processed systems. In term of speed it should be noticed that the same technological progress that provides ever-increasing computation speeds also permits increased data acquisition rates. Then it is clear that computation time remains the bottleneck of methods based on numeric processing of Ascans. In this context, the development of an all-optical method is obviously of scientific interest. Concerning the number of resolved points in the wavelength-depth space in the case of the scan-free system, the number of resolved points is directly related to the total number of pixels of the two-dimensional detector. To achieve the same resolution in TDOCT a much higher number of acquired points will be required, which will slow down the acquisition rate.

Tunable inherent dispersion compensation (figures and text are extracted or inspired from [55] by Froehly et al). In 1997 Tearney introduced a rapid scanning optical delay line based on a diffraction grating as a tool for high speed and long depth range acquisition in TDOCT [24]. In 2002 Smith showed the capability of such a delay line to introduce depth dependent dispersion compensation in TDOCT [63] using the high order terms of diffraction gratings as already known in temporal pulse shaping. Time domain scan-free methods based on diffraction gratings have the same properties. In 2009 Froehly demonstrated the capability of such a system to realize tunable dispersion compensation [55]. The principle is shown in figure 9. The main idea is that different wavelengths diffracted by the grating will reach a plane at a distance $d$ from the grating (see figure 9(a)) at different times due to the fact that they are diffracted at different angles. Then the expression of the correlogram when instead of imaging the grating plane we image a plane at a distance $d$ from the grating becomes

$$
\begin{aligned}
C(z) & =I_{0}+2 \mathcal{R} e\left[\int_{\nu} R(\nu) \hat{S}(\nu) \mathrm{e}^{-\mathrm{j} 2 \pi\left(\frac{z}{\gamma c}\left(2 \sin \theta_{i} \cos \phi\right)\right) \nu} \mathrm{e}^{\mathrm{j} \frac{2 \pi z}{\gamma \Lambda}}\right. \\
& \left.\times \mathrm{e}^{\mathrm{j} \frac{2 \pi}{\lambda}\left(\sqrt{1-\left(-\frac{\lambda}{\Lambda}+\sin \left(\theta_{i}-\phi\right)\right)^{2}}-\cos \left(\theta_{i}+\phi\right)\right) d} \mathrm{~d} \nu\right]
\end{aligned}
$$

In this equation the phase term written $\frac{2 \pi}{\lambda}\left(\sqrt{1-\left(-\frac{\lambda}{\Lambda}+\right.}\right.$ $\left.\overline{\left.\sin \left(\theta_{i}-\phi\right)\right)^{2}}-\cos \left(\theta_{i}+\phi\right)\right) d$ is proportional to $d$ and is nonlinear with the light frequency. This term is responsible for group velocity dispersion as well as for higher order dispersion terms. In the paper the dispersion compensation of a glass plate of $9 \mathrm{~mm}$ up to the diffraction limit has been shown for a bandwidth of $100 \mathrm{~nm}$ (see figure 10). The possibility of static depth dependent dispersion compensation has been suggested.

\section{Conclusion and outlook}

Short pulse measurement systems and nonlinear optical correlators have strongly influenced the new high resolution optical biological imaging techniques. OCT, since its initial demonstration almost 20 years ago, has then widely spread in the field of non-destructive and non-invasive high resolution imaging especially in ophthalmology. Speed, sensitivity, and resolution have reached tremendous performance thanks particularly to the FDOCT system. Numerous commercial 


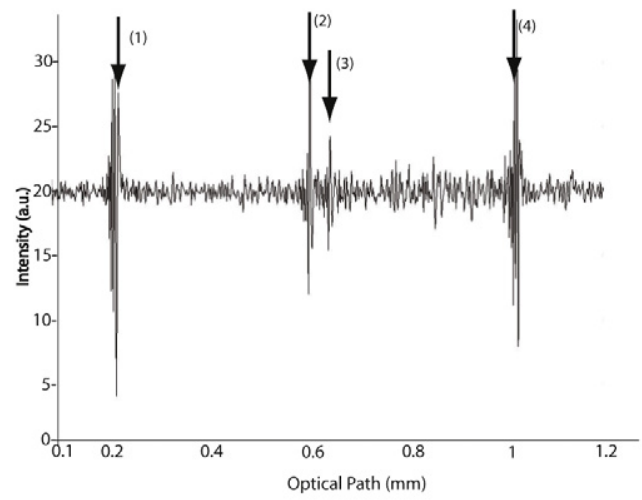

(a)

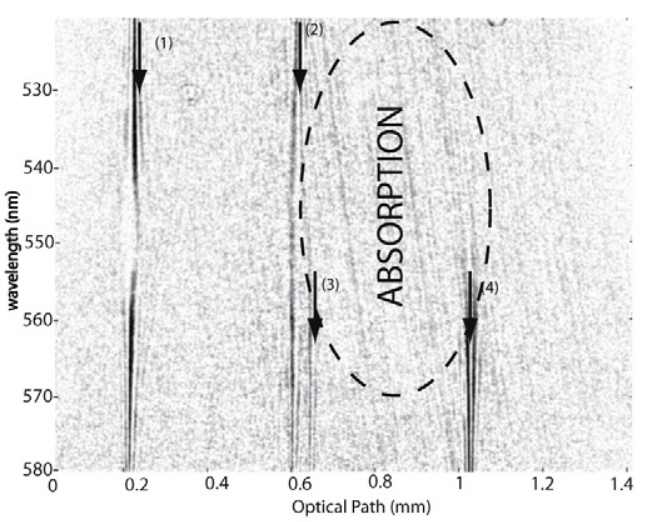

(b)

Figure 8. Experimental OCT signals obtained on an eosin solution layer sandwiched by capillarity between two microscope cover-slides. (a) Classical 'A-scan' obtained with our system (without scanning). (b) Instantaneous spectro-tomographic signal with visible depth resolved spectral absorption of the eosin layer. (Reprinted from [54] by Froehly et al.)

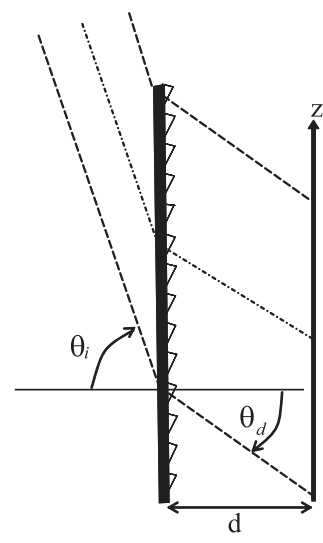

(a)

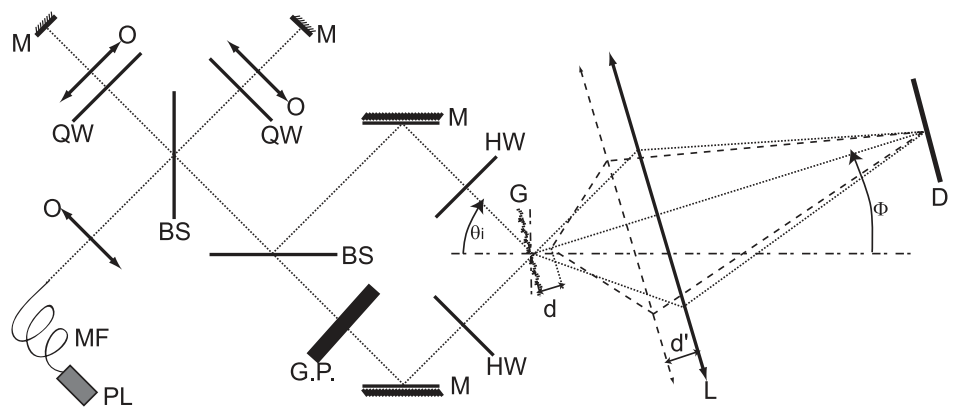

(b)

Figure 9. Parameters (a) and experimental setup (b) for dispersion compensation: M, mirrors; BS, polarizing beam splitter cube; QW, quarter wave plate; HW, half wave plate; L, spherical lens; PL, pump laser; MF, microstructured optical fiber; D, CCD detector; G, transmission diffraction grating; O, microscope objectives; GP, glass plates. (Reprinted from [55] by Froehly et al.)

systems have already reached the diagnostic market in ophthalmology, where it has already become a standard.

Time domain scan-free correlation systems, widely used in the field of pulse shape measurements, are still less common and widely unknown, in the field of biological imaging, and do not yet have commercial developments. This is mainly due to the fact that they, in principle, share the same sensitivity limitations with 'former' TDOCT. Clearly this limitation is critical when sensitivity and real-time conditions have to be both fulfilled (this is especially the case in eye imaging, when eye safety standards impose a low incident power). Nevertheless, authors have recently shown high speed imaging capability together with quite good sensitivity in in vivo biological media [56, 52]. Furthermore, specific properties of these systems, such as dispersion compensation, optical processing of Wigner functions, potentially depth dependent dispersion compensation and artifact-free optically processed time domain imaging, are also highly attractive and unique features. Such properties need to be further confirmed and demonstrated for imaging of biological samples. First preliminary results for biomedical imaging with good sensitivity are encouraging. Such systems may become a standard for functional imaging in some media when real-time access to functional information is more important than the sensitivity (i.e. less scattering samples and/or fewer constraints in incident light power). Then the 'post-processing'-free (as compared to FDOCT) and scan-free (as compared to TDOCT) properties become a key point especially in the frame of spectroscopic OCT. This will need to be further demonstrated in proper applications. Functional imaging of an in vitro epidermal layer of excised skin or skin models can be one of them: the 'real-time' monitoring of pharmaceutical drugs introduced in the superficial skin layer is of special interest for the pharmaceutical market. So far there is no database concerning such tests. Results could then be compared with FDOCT outcomes and also the standard biopsy. Nonbiological imaging is also another potential application of such systems (real-time monitoring of laser ablation process is already a matter of research in OCT but the spectroscopic feature is not yet implemented as it normally needs postprocessing). To have a complete overview of the impact of scan-free systems in the field of biological imaging the 


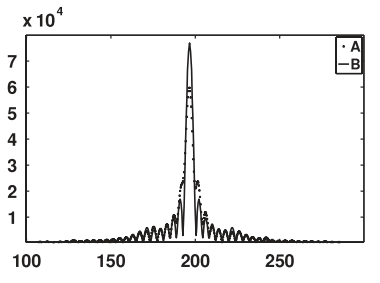

(a1)

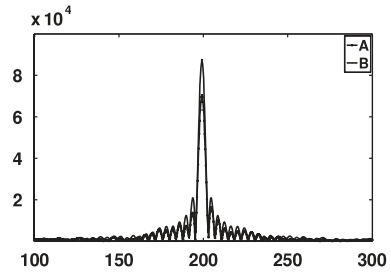

(a2)

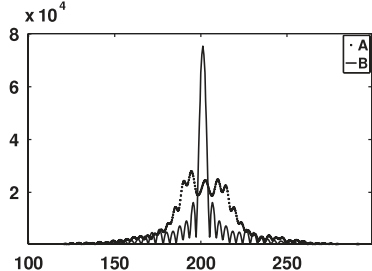

(b1)

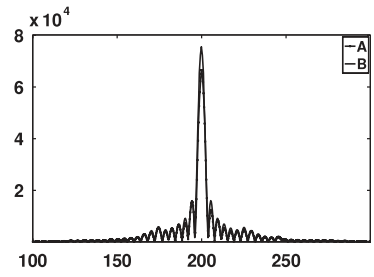

(b2)

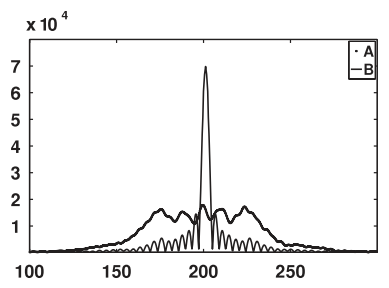

(c1)

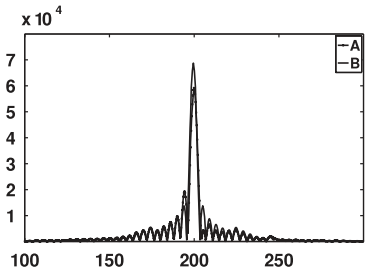

(c2)

Figure 10. Experimental dispersion compensation: (a1), (b1), (c1) are obtained for respectively 1, 4, 9 mm glass thicknesses without dispersion correction whereas (a2), (b2), (c2) are correlations obtained for the same glass thicknesses but with a dispersion correction due to an image plane shift $(d)$ of respectively $-0.29,-1,-2.25 \mathrm{~mm}$. In each case curve (A) is the autocorrelation that allows the comparison with the ideal point spread function (PSF). (Reprinted from [55] by Froehly et al.)

complexity of the system with respect to obtained results has also to be addressed as a major go/no go criterion. Indeed, FDOCT techniques demonstrate impressive images in term of speed, resolution and functionalities but often at the cost of complexity and price, whereas scan-free systems, with of course an inherent lower sensitivity, show globally simpler architectures.

\section{References}

[1] Gu M 2000 Advanced Optical Imaging Theory (Springer Series in Optical Science) (Berlin: Springer)

[2] Diels J-C M, Fontaine J J, McMichael I C and Simoni F 1985 Control and measurement of ultrashort pulse shapes (in amplitude and phase) with femtosecond accuracy Appl. Opt. 24 1270-82

[3] Trebino R 2002 Frequency-resolved Optical Gating: The Measurement of Ultrashort Laser Pulses (Dordrecht: Kluwer Academic)

[4] Kane D J and Trebino R 1993 Single-shot measurement of the intensity and phase of an arbitrary ultrashort pulse by using frequency-resolved optical gating Opt. Lett. 18 823-5

[5] Huang D et al 1991 Optical coherence tomography Science 2541178

[6] Zeylikovich I and Alfano R R 1996 Ultrafast dark-field interferometric microscopic reflectometry $\mathrm{Opt}$. Lett. 21 1682-4

[7] Fercher A F 1990 Ophthalmic Interferometry in Optics in Medicine, Biology and Environmental Research ed $\mathrm{G}$ von Bally and S Khanna (Amsterdam: Elsevier) pp 221-35

[8] Hitzenberger C K 1991 Optical measurement of the axial eye length by laser Doppler interferometry Invest. Ophthalmol. Vis. Sci. 32 616-24

[9] Fercher A F, Hitzenberger C K, Drexler W, Kamp G and Sattmann H 1993 In vivo optical coherence tomography Am. J. Ophthalmol. 116 113-4

[10] Swanson E A, Izatt J A, Hee M R, Huang D, Lin C P, Schuman J S, Puliafito C A and Fujimoto J G 1993 In vivo retinal imaging by optical coherence tomography Opt. Lett. $181864-6$
[11] Purchase K, Brady D and Wagner K 1993 Time-of-flight cross correlation on a detector array for ultrafast packet detection Opt. Lett. 18 2129-31

[12] Zeylikovich I, Liu Q, Bai G, Zhadin N, Gorokhovsky A and Alfano R R 1995 Interferometric 2D imaging amplitude correlator for ultrashort pulses Opt. Commun. 115 485-90

[13] Saleh B E A and Teich M C 1991 Fundamentals of Photonics (New York: Wiley-Interscience)

[14] Bourquin S 2000 Low-coherence interferometry based on customized detector arrays PhD Thesis EPFL, no 2171

[15] Leitgeb R, Hitzenberger C K and Fercher A F 2003 Performance of fourier domain vs. time domain optical coherence tomography Opt. Express 11 889-94

[16] Flournoy P A, McClure R W and Wyntjes G 1972 White-light interferometric thickness gauge Appl. Opt. 11 1907-15

[17] Youngquist R C, Carr S and Davies D E N 1987 Optical coherence-domain reflectometry: a new optical evaluation technique Opt. Lett. 12 158-60

[18] Clivaz X, Marquis-Weible F, Salathé R P, Novàk R P and Gilgen H H 1992 High-resolution reflectometry in biological tissues Opt. Lett. 17 4-6

[19] Fontaine J J, Diels J-C, Wang C-Y and Sallaba H 1981 Subpicosecond-time-domain reflectometry Opt. Lett. 6 405-7

[20] Fujimoto J G, Silvestri S D, Ippen E P, Puliafito C A, Margolis R and Oseroff A 1986 Femtosecond optical ranging in biological systems Opt. Lett. 11 150-2

[21] Morgner U, Drexler W and Fujimoto J G 1999 In vivo ultrahigh-resolution optical coherence tomography Opt. Lett. 24 1221-3

[22] Hartl I, Li X D, Chudoba C, Ghanta R K, Ko T H, Fujimoto J G, Ranka J K and Windeler R S 2001 Ultrahigh-resolution optical coherence tomography using continuum generation in an air-silica microstructure optical fiber Opt. Lett. 26 608-10

[23] Kwong K F, Yankelevich D, Chu K C, Heritage J P and Dienes A $1993400 \mathrm{~Hz}$ mechanical scanning optical delay line Opt. Lett. 18 558-60

[24] Tearney G J, Bouma B E and Fujimoto J G 1997 High-speed phase- and group-delay scanning with a grating-based phase control delay line Opt. Lett. 22 1811-3

[25] Tearney G J, Brezinski M E, Bouma B E, Boppart S A, Pitris C, Southern J F and Fujimoto J G 1997 In vivo endoscopic optical biopsy with optical coherence tomography Science 2762037 
[26] Fercher A F, Drexler W, Hitzenberger C K and Lasser T 2003 Optical coherence tomography-principles and applications Rep. Prog. Phys. 66 239-303

[27] Bouma B E and Tearney G J Handbook of Optical Coherence Tomography (New York: Dekker)

[28] Drexler W and Fujimoto J G 2008 Optical Coherence Tomography: Technology and Applications (Berlin: Springer)

[29] Fercher A F, Hitzenberger C K, Kamp G and El-Zaiat S Y 1995 Measurement of intraocular distances by backscattering spectral interferometry Opt. Commun. 117 43-8

[30] Froehly C, Lacourt A and Viénot J C 1973 Notions de réponse impulsionnelle et de fonction de transfert temporelles des pupilles optiques, justifications expérimentales et applications Nouv. Rev. Opt. 4 183-96

[31] Viénot J C, Goedgebuer J P and Lacourt A 1977 Space and time variables in optics and holography: recent experimental aspects Appl. Opt. 16 454-61

[32] Lepetit L, Chériaux G and Joffre M 1995 Linear techniques of phase measurement by femtosecond spectral interferometry for applications in spectroscopy J. Opt. Soc. Am. B 12 2467-74

[33] Iaconis C and Walmsley I 1998 Spectral phase interferometry for direct electric-field reconstruction of ultrashort optical pulses Opt. Lett. 23 792-4

[34] Wojtkowski M, Kowalczyk A, Leitgeb R and Fercher A F 2002 Full range complex spectral optical coherence tomography technique in eye imaging Opt. Lett. 27 1415-7

[35] Yasuno Y, Makita S, Endo T, Aoki G, Sumimura H, Itoh M and Yatagai T 2004 One-shot-phase-shifting Fourier domain optical coherence tomography by reference wavefront tilting Opt. Express 12 6184-91

[36] Yasuno Y, Makita S, Endo T, Aoki G, Itoh M and Yatagai T 2006 Simultaneous B-M-mode scanning method for real-time full-range Fourier domain optical coherence tomography Appl. Opt. 45 1861-5

[37] Bachmann A, Leitgeb R and Lasser T 2006 Heterodyne Fourier domain optical coherence tomography for full range probing with high axial resolution Opt. Express 14 1487-96

[38] Wang R K 2007 In vivo full range complex Fourier domain optical coherence tomography Appl. Phys. Lett. 90054103

[39] Leitgeb R A, Hitzenberger C K, Fercher A F and Bajraszewski T 2003 Phase-shifting algorithm to achieve high-speed long-depth-range probing by frequency-domain optical coherence tomography Opt. Lett. 28 2201-3

[40] Leitgeb R A, Michaely R, Lasser T and Sekhar S C 2007 Complex ambiguity-free Fourier domain optical coherence tomography through transverse scanning Opt. Lett. 32 3453-5

[41] Baumann B, Pircher M, Gotzinger E and Hitzenberger C K 2007 Full range complex spectral domain optical coherence tomography without additional phase shifters Opt. Express 15 13375-87

[42] Bu P, Wang X and Sasaki O 2008 One-shot parallel complex Fourier-domain optical coherence tomography using a spatial carrier frequency Opt. Eng. 47050502

[43] Ustun T E, Iftimia N V, Ferguson R D and Hammer D X 2008 Real-time processing for Fourier domain optical coherence tomography using a field programmable gate array Rev. Sci. Instrum. 79114301

[44] Watanabe Y and Itagaki T 2009 Real-time display on Fourier domain optical coherence tomography system using a graphics processing unit J. Biomed. Opt. 14060506
[45] Connes P 1957 Un nouveau type de spectromètre: l'interferomètre réseaux J. Mod. Opt. 4 136-44

[46] Verrier I, Brun G, Barthelemy A, Froehly C and Goure J P 1992 Measurements of mode propagation time in multimode fibers using a real time interferometric amplitude correlator J. Opt. Commun. 13 134-9

[47] Verrier I, Brun G and Goure J P 1997 SISAM interferometer for distance measurements Appl. Opt. 36 6225-30

[48] Zeylikovich I and Alfano R R 1997 Ultrafast correlation interferometric imaging through a moving scattering medium Opt. Commun. 135 217-22

[49] Zeylikovich I, Gilerson A and Alfano R R 1998 Nonmechanical grating-generated scanning coherence microscopy Opt. Lett. 23 1797-9

[50] Hauger M W C and Hellmuth T 2003 Interferometer for optical coherence tomography Appl. Opt. 42 3896-902

[51] Koch P, Hellemanns V and Hüttmann G 2006 Linear optical coherence tomography system with extended measurement range Opt. Lett. 31 2882-4

[52] Watanabe Y, Sajima F, Itagaki T, Watanabe K and Shuto Y 2009 High-speed linear detection time domain optical coherence tomography with reflective grating-generated spatial reference delay Appl. Opt. 48 3401-6

[53] Froehly L, Ouadour M, Petitjean G, Furfaro L, Sandoz P, Gharbi T, Leproux P, Huss G and Couderc V 2006 Real-time optical coherence spectrotomography: proof of principle Proc. SPIE 6191 173-82

[54] Froehly L, Ouadour M, Furfaro L, Sandoz P, Gharbi T, Leproux P, Huss G and Couderc V 2008 Spectroscopic OCT by grating-based temporal correlation coupled to optical spectral analysis Int. J. Biomed. Imaging 2008752340

[55] Froehly L, Furfaro L, Sandoz P and Jeanningros P 2009 Dispersion compensation properties of grating-based temporal-correlation optical coherence tomography systems Opt. Commun. 282 1488-95

[56] Watanabe Y, Yamada K and Sato M 2006 Three-dimensional imaging by ultrahigh-speed axial-lateral parallel time domain optical coherence tomography Opt. Express 14 5201-9

[57] Izatt J A, Kulkarni M D, Yazdanfar S, Barton J K and Welch A J 1997 In vivo bidirectional color Doppler flow imaging of picoliter blood volumes using optical coherence tomography Opt. Lett. 22 1439-41

[58] Schmitt J M, Xiang S H and Yung K M 1998 Differential absorption imaging with optical coherence tomography J. Opt. Soc. Am. A 15 2288-96

[59] Morgner U, Drexler W, Kärtner F X, Li X D, Pitris C, Ippen E P and Fujimoto J G 2000 Spectroscopic optical coherence tomography Opt. Lett. 25 111-3

[60] Watanabe W and Itoh K 2000 Coherence spectrotomography: optical spectroscopic tomography with low-coherence interferometry Opt. Rev. 7 406-14

[61] Laubscher M, Bourquin S, Froehly L, Karamata B and Lasser T 2004 Spectroscopic optical coherence tomography based on wavelength de-multiplexing and smart pixel array detection Opt. Commun. 237 275-83

[62] Xu C, Vinegoni C, Ralston T S, Luo W, Tan W and Boppart S A 2006 Spectroscopic spectral-domain optical coherence microscopy Opt. Lett. 31 1079-81

[63] Smith E D J, Zvyagin A V and Sampson D D 2002 Real-time dispersion compensation in scanning interferometry $O p t$. Lett. 27 1998-2000 